\title{
THE SIGNIFICANCE OF CLAUDIN-1 AND S100A4 EXPRESSION IN PRIMARY COLORECTAL CARCINOMA
}

\author{
Randa Kaf, Essam Saba, Wafaa Refaat, Amira Bahgat \\ Pathology Department, Faculty of Medicine, Zagazig University, Egypt
}

\begin{abstract}
Background: Metastasis is a major cause of death in colorectal carcinoma (CRC). Epithelial to mesenchymal transition (EMT) plays a role in promoting carcinoma invasion and metastasis. Many molecules are crucial in EMT, including claudin-1 and S100A4.
\end{abstract}

Aim of work: The aim of this study is to assess the pattern of expression and the prognostic significance of claudin-1 and S100A4 in primary colorectal carcinoma.

Methods: Claudin-1 and S100A4 expressions were retrospectively analysed by immunohistochemistry in 55 paraffin-embedded specimens of patients with primary colorectal carcinoma. The expressions were correlated with clinicopathological variables, Dukes staging and lymph node metastasis.

Results: Normal colonic and rectal mucosa exhibited diffuse, strong circumferential linear membranous pattern of claudin-1 staining (score 0$)$. Twenty six cases $(65 \%)$ of primary colorectal carcinomas showed decreased claudin1 expression compared to normal mucosa and 14 cases (35\%) showed similar claudin 1 expression (score 0 ). None of the studied cases showed increased claudin 1 expression. A highly statistically significant relationship was found between claudin1 expression and Lymphovascular invasion ( $\mathrm{p}=0.003$ ), degree of lymphocytic infiltration, peritumoral budding, lymph node ratio, lymph node metastasis and Dukes staging $(\mathrm{p}<0.001)$. Fourteen cases $(35 \%)$ showed negative S100A4 expression (score 0-2), 10 cases (25\%) showed mild positive S100A4 expression (score 3), 9 cases (22.5\%) showed moderate positive S100A4 expression (score 4-5) and 7 cases $(17.5 \%)$ showed marked positive S100A4 expression (score 6). A highly statistically significant relationship was found between S100A4 expression and grade of differentiation, lymphovascular invasion $(\mathrm{p}=0.002)$, lymphocytic infiltration, peritumoral budding, lymph node metastasis, lymph node ratio and Dukes staging ( $\mathrm{p}<0.001)$. By Pearson's correlation coefficient $(\mathrm{r})$ method, there is a strong inverse correlation between claudin1 and S100A4 expression.

Conclusions: Loss of tight junctions represented by decreased claudin1 expression and acquisition of mesenchymal properties represented by S100A4 overexpression are important in primary colorectal carcinoma progression and metastasis.

Keywords: Primary colorectal carcinoma; S100A4; Claudin-1; EMT.

\begin{tabular}{ll}
\hline Corresponding author: Randa Mohamed Kaf & Received: November 2015 \\
Mobile: 01004722434 & \\
e.mail: trjn2010@yahoo.com & Accepted: January 2016 \\
\hline
\end{tabular}

\section{INTRODUCTION}

C olorectal carcinoma (CRC) is the third most common cancer, and is a leading cause of death worldwide. ${ }^{(1)}$ According to the Egyptian National Cancer Institute, CRC carcinoma in Egypt contributes $6.5 \%$ of all cancers. $^{(2)}$

Invasion and metastasis are two important biological characters of malignant tumor. The five-year survival of CRC is closely related to the invasive and metastatic ability according to Dukes staging. ${ }^{(3)}$

One major molecule that is linked to metastasis formation is overexpression of S100 calcium binding protein A4 (S100A4), which was originally identified as metastasin 1 (MTS1). (4) S100A4 regulates many cellular processes needed for metastasis formation such as regulation of angiogenesis, motility, invasion, and cell survival. ${ }^{(5)}$ The expression of S100A4 is known to be increased in CRC cells. (6) The elevated concentration of S100A4 within the tumor tissue is indicative of tumor progression and lymph node metastasis with subsequent poor prognosis ${ }^{(7)}$ and worse survival in patients with CRC. ${ }^{(8)}$ It provides a marker for the early identification of patients at high risk for distant metastasis. ${ }^{(9)}$ 
During cancer development/ progression, tight junctions are often lost, or claudins translocate to other cellular compartments. This facilitates malignant transformation and epithelial-mesenchymal transition (EMT). ${ }^{(10)}$

Expression of claudin-1 has shown to be decreased in breast, pancreas and colorectal carcinomas. However, there are upregulation of claudin-1 in pancreatic and thyroid carcinoma. (11)

This study aims to assess pattern of expression and prognostic significance of claudin-1 and S100A4 in primary colorectal carcinoma.

\section{MATERIAL AND METHODS}

Fifty five formalin fixed paraffin embedded tissue blocks of primary colorectal carcinoma cases and concordant cancer adjacent normal mucosa were retrieved from the archives of the Departments of Pathology, Faculty of Medicine, Zagazig University, Mansoura University and National Cancer Institute between the years of 2010 and 2012 . Clinicopathological informations were abstracted from archive files of the corresponding departments. Only primary colorectal carcinoma cases were included in this study. Recurrent cases, cases without lymph node reporting and cases who received preoperative adjuvant chemotherapy or radiotherapy were excluded. All tissue samples were obtained through surgical resection of colon $(n=35)$ and rectum $(n=20)$ and fixed in $10 \%$ formalin.

Typing and grading of colorectal carcinomas were based on WHO 2010 published criteria. ${ }^{(12)}$ Adenocarcinoma (NOS) was divided into well (grade 1), mod

erately (grade 2) and poorly differentiated (grade 3). Mucinous adenocarcinoma and signet ring cell carcinoma were considered poorly differentiated (grade 3). ${ }^{(13)}$ Staging of colorectal carcinomas was defined according to the Dukes' staging system. ${ }^{(14)}$

Peritumoral budding was assessed semiquantitatively on $\mathrm{H} \& \mathrm{E}$ stained sections. The tumor border was scanned at 10x power and the area of most dense budding was identified. In the center of this area, tumor buds (single cells or clusters of up to 5 cells) were counted at 20xmagnification. ${ }^{(15)}$ Cases were then divided into two groups: counts of
0-9 tumor buds were classified as low-grade, while counts of $\geq 10$ tumor buds were classified as high-grade budding. ${ }^{(16)}$

Patients were then assigned into two groups, based on their LNR: LNR1, less than or equal 0.25 ; LNR2: greater than $0.25 .^{(17)}$ Lymphocytic infiltration was assessed and divided into mild, moderate and severe. Lymphovascular invasion was assessed whether present or absent. ${ }^{(8)}$

\section{Immunohistochemistry}

Immunohistochemical staining was carried out using indirect streptoavidin-biotin immunoperoxidase technique. Tissue sections (3-5 $\mu \mathrm{m})$ were deparaffinized in xylene and rehydrated in graded alcohol. Slides were incubated for 10 minutes in $0.3 \%$ hydrogen peroxide in absolute methanol to block endogenous peroxidase activity. Antigen retrieval was performed using Dako target retrieval solution ( $\mathrm{pH}$ 6.0) (Dako, CA, USA) .The slides were then incubated for 60 minutes at room temperature using a rabbit polyclonal anti-claudin-1 antibody (Lab Vision, Corp, Fremont, CA, USA), with a 1:50 dilution; and a rabbit polyclonal anti S100A4 antibody (Lab Vision, Corp, Fremont, CA, USA), with a 1:200 dilution, then washed with two changes of PBS, stained again with secondary antibody for 15 minutes at room temperature, then rinsed in the buffer again.

Positive and negative controls were stained at the same setting with the selected slides.

\section{Immunohistochemical Evaluation}

A reaction was considered claudin-1 positive if any number of tumor cells showed membranous and/or cytoplasmic positivity. For scoring both the intensity and the extent of claudin-1 immunostaining were evaluated in tumor tissue as well as in the concordant cancer- adjacent normal mucosa which served as an internal control. The intensity of membranous /or cytoplasmic staining was graded as either weak $=1$; moderate $=2$; or strong $=3$. The extent of staining was evaluated semi quantitatively and categorized as focal $(<10 \%)=1$; regional $(11-50 \%)=2$; or diffuse $(>50 \%)=3$.

Total scores for caludin-1 expression were then calculated by multiplying the obtained scores of both intensity and extent of staining. 
(18) Total scores of claudin-1 expression in the studied colorectal carcinoma slides were compared to the adjacent normal mucosa and a final computed score for each case was calculated by subtraction of the total score in normal mucosa from the total score in the tumor. ${ }^{(18)}$

In cases where total scores for claudin-1 expression in the tumor were equal to the adjacent normal mucosa, the tumor was designated as having similar claudin-1 expression to normal mucosa and classified as score 0 . If the total score for claudin-1 expression in the tumor was less than that in the normal mucosa, the tumor was designated as having decreased claudin- 1 expression and the final score less than 0 . If the total claudin-1 staining score in the tumor was higher than the normal mucosa, the tumor was designated as having increased claudin- 1 expression and the final score is more than $0 .{ }^{(18)}$

S100A4 expressed in cytoplasm. The extent of staining was categorized as $0:<5 \%$; $1: \geq 5-25 \% ; 2: 25 \%-50 \% ; \quad 3:>50 \%$. The intensity of staining was graded as either: Negative $=0$; weak $=1$; moderate $=2$; or strong $=3$. Final result combines the scores from the two parameters (ranging from 0-6). Scores of 0-2 were considered as negative expression while scores of 3-6 were defined as positive expression. ${ }^{(19)}$

\section{Statistical analysis}

All data were collected, tabulated and statistically analyzed using SPSS 20 for windows (IBM Corp .Armonk. 2011). Continuous quantitative variables e.g. age were expressed as the mean \pm SD \& median (range), and categorical qualitative variables were expressed as an absolute frequencies "number" $\&$ relative frequencies (percentage). Categorized data were compared using the Chi-square $(\chi 2)$ test while Fisher's Exact test was used if expected count $<5$. Inter-rater agreement (kappa) in correlation between Dukes and TNM staging was calculated. Pearson's correlation coefficient (r) was calculated to find the closeness and relation between claudin 1 and S100A4 expression.

All tests were two sided. $\mathrm{P}$ value $<0.05$ was considered statistically significant (S) and $\mathrm{p}$ value $>0.05$ was considered non statistically significant (NS).

\section{RESULTS}

Forty cases were fitting into the selection criteria: of the 15 cases excluded, for 10 cases there was no lymph node reporting, in 3 cases the tumor was recurrent and 2 cases received preoperative chemotherapy. Clinicopathologic characteristics and staging data of primary colorectal carcinoma cases are summarized in Table 1.

The mean age was $49.7 \pm 11.39$ years (range 23-69), 23 males and 17 females were included in the study. Adenocarcinoma (NOS) is the commonest histologic type representing 31 cases $(77.5 \%)$, followed by mucinous carcinoma representing 7 cases $(17.5 \%)$ then signet-ring carcinoma representing 2 cases (5\%). Most of the tumors 27/40 (67.5\%) had a tumor size $\leq 5 \mathrm{~mm}$, whereas $13 / 40(32.5 \%)$ had a tumor size $>5 \mathrm{~mm}$. Histologic grading showed 17 cases $(42.5 \%)$ with grade 1 (well differentiated), 13 cases $(32.5 \%)$ with grade 2 (moderately differentiated) and 10 cases $(25 \%)$ with grade 3 (poorly differentiated) adenocarcinomas. Evidence of lymphovascular invasion was present only in 12 cases $(30 \%)$. As for neural invasion, it was present in 5 cases $(12.5 \%)$. Most of the tumors 18/40 $(45 \%)$ showed peritumoral moderate lymphocytic infiltration. High grade peritumoral budding was present in 23 cases (57.5\%), while 17 cases $(42.5 \%)$ showed low grade peritumoral budding. The mean of lymph node ratio (LNR) is $0.18 \pm 0.2$, range ( 0 $-1)$. Evidence of lymph node metastasis was detected in 22 cases $(55 \%)$. The harvested lymph nodes ranged from 3-40.Three cases $(7.5 \%)$ were diagnosed at Dukes A equivalent to TNM stage I. 15 cases $(37.5 \%)$ at stage Dukes B / TNM II. 18 cases $(45 \%)$ at stage Dukes C /TNM III and 4 cases $(10 \%)$ at stage Dukes D/ TNM IV. 
Table 1 The clinical and pathological characteristics of primary colorectal carcinoma cases

Variables

Age

Mean \pm SD

Sex

Male

Female

Histological type

\begin{tabular}{|c|c|}
\hline Adenocarcinoma (NOS) & $31(77.5 \%)$ \\
\hline Mucinous & $7(17.5 \%)$ \\
\hline Signet ring & $2(5 \%)$ \\
\hline \multicolumn{2}{|l|}{ Tumor size } \\
\hline$\leq 5 \mathrm{~cm}$ & $27(67.5 \%)$ \\
\hline$<5 \mathrm{~cm}$ & $13(32.5 \%)$ \\
\hline \multicolumn{2}{|l|}{ Grading } \\
\hline Grade I & $17(42.5 \%)$ \\
\hline Grade II & $13(32.5 \%)$ \\
\hline Grade III & $10(25 \%)$ \\
\hline \multicolumn{2}{|l|}{ Lymphovascular invasion } \\
\hline Absent & $28(70 \%)$ \\
\hline Present & $12(30 \%)$ \\
\hline \multicolumn{2}{|l|}{ Neural invasion } \\
\hline Absent & $35(87.5 \%)$ \\
\hline Present & $5(12.5 \%)$ \\
\hline \multicolumn{2}{|l|}{ Lymphocytic infiltration } \\
\hline Mild & $11(27.5 \%)$ \\
\hline Moderate & $18(45 \%)$ \\
\hline Severe & $11(27.5 \%)$ \\
\hline \multicolumn{2}{|l|}{ peritumoral budding } \\
\hline Low & $17(42.5 \%)$ \\
\hline High & $23(57.5 \%)$ \\
\hline \multicolumn{2}{|l|}{$\mathrm{LN}$ ratio (LNR) } \\
\hline Mean \pm SD & $0.18 \pm 0.23$ \\
\hline $\begin{array}{l}\text { Median (Range) } \\
\text { Cut off point }\end{array}$ & $\begin{array}{c}0.11(0-1) \\
0.25\end{array}$ \\
\hline \multicolumn{2}{|l|}{ Lymph node metastasis } \\
\hline Negative & $18(45 \%)$ \\
\hline Positive & $22(55 \%)$ \\
\hline $\begin{array}{l}\text { Dissected lymph nodes } \\
\text { Mean } \pm \text { SD } \\
\text { Median (range) }\end{array}$ & $\begin{array}{c}15 \pm 8.58 \\
14(3-40) \\
\end{array}$ \\
\hline $\begin{array}{c}\text { Dukes staging } \\
\text { Stage A } \\
\text { Stage B } \\
\text { Stage C } \\
\text { Stage D }\end{array}$ & $\begin{array}{c}3(7.5 \%) \\
15(37.5 \%) \\
18(45 \%) \\
4(10 \%)\end{array}$ \\
\hline
\end{tabular}

\section{colorectal carcinoma cases $(n=40)$}

$49.68 \pm 11.39$, range $(23-69)$

$23(57.5 \%)$

$17(42.5 \%)$ 


\section{Claudin1 expression:}

Normal colonic and rectal mucosa exhibited diffuse, strong circumferential linear membranous pattern of staining (score 0) (Figure 1). The fourteen colorectal cases that showed similar pattern of staining to normal adjacent mucosa was scored 0 and claudin 1 expression showed no loss (Figure 2). A fragmented and weaker circumferential linear pattern was interpreted as decreased claudin1 expression and staining scores ranged from-3 to -8 (Figures 3\&4). Overall, 65\% (26/40) of colorectal carcinoma cases showed decreased claudin 1 expression and 14 cases (35\%) showed similar claudin1 expression (score 0). None of the studied cases showed increased claudin1 expression. Analysis of claudin1 expression with clinicopathologic characteristics was presented in Table 2.

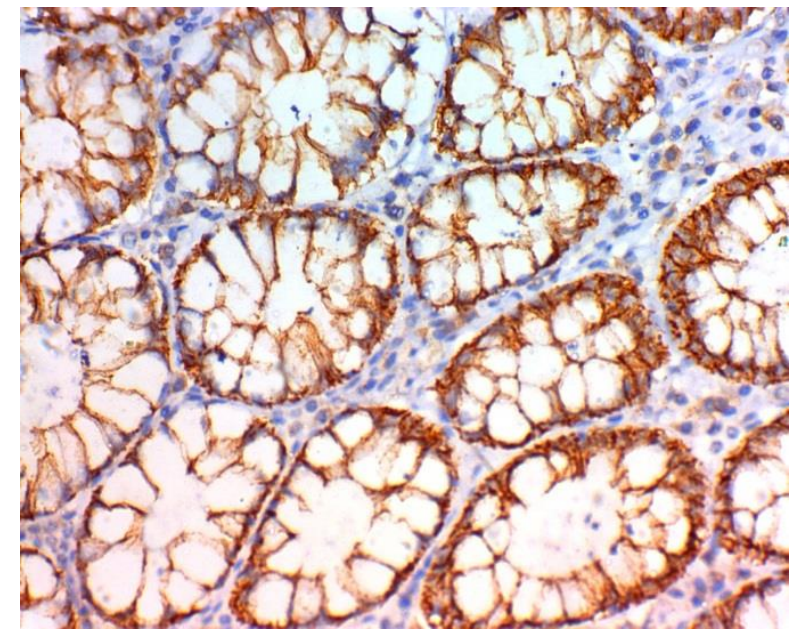

Figure 1 Normal colonic mucosa showing complete linear membranous staining for Claudin1 (Immunoperoxidase staining, DAB chromogen, Mayer's hematoxyline counter stain, original magnification, X400)

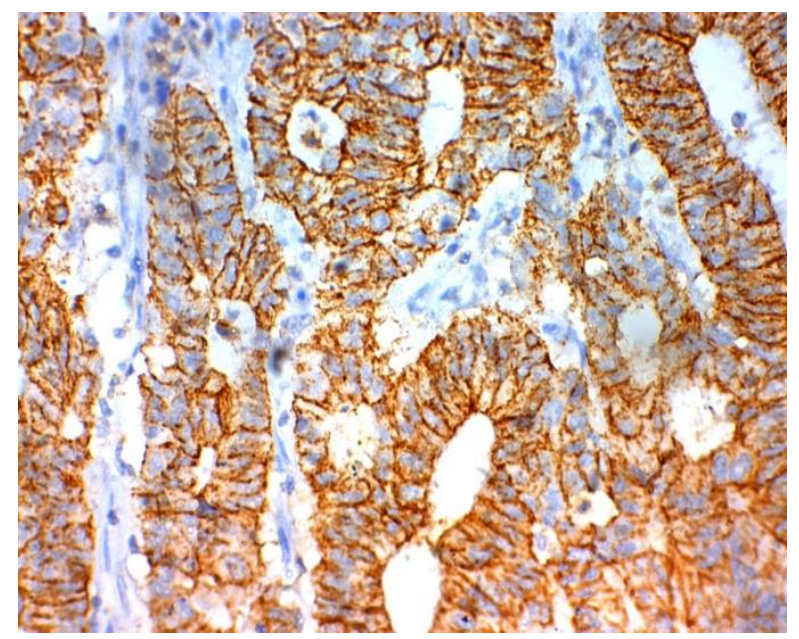

Figure 3 Colorectal adenocarcinoma grade II showing moderate, incomplete linear membranous claudin1 staining, score -3 (decreased expression) (Immunoperoxidase staining, DAB chromogen, Mayer's hematoxyline counter stain, original magnification, X400).

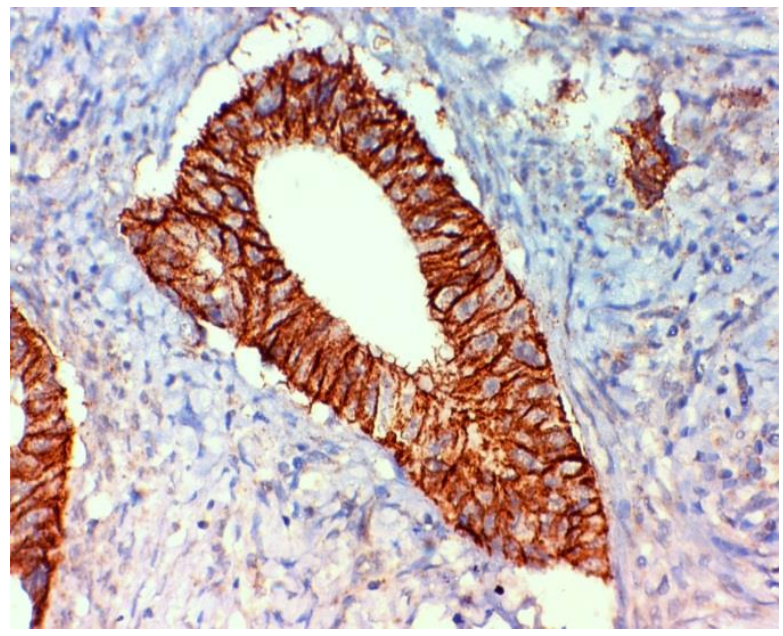

Figure 2 Colorectal adenocarcinoma grade I showing strong, complete linear membranous claudin 1 staining, score 0 (similar expression) (Immunoperoxidase staining, DAB chromogen, Mayer's hematoxyline counter stain, original magnification, X400)

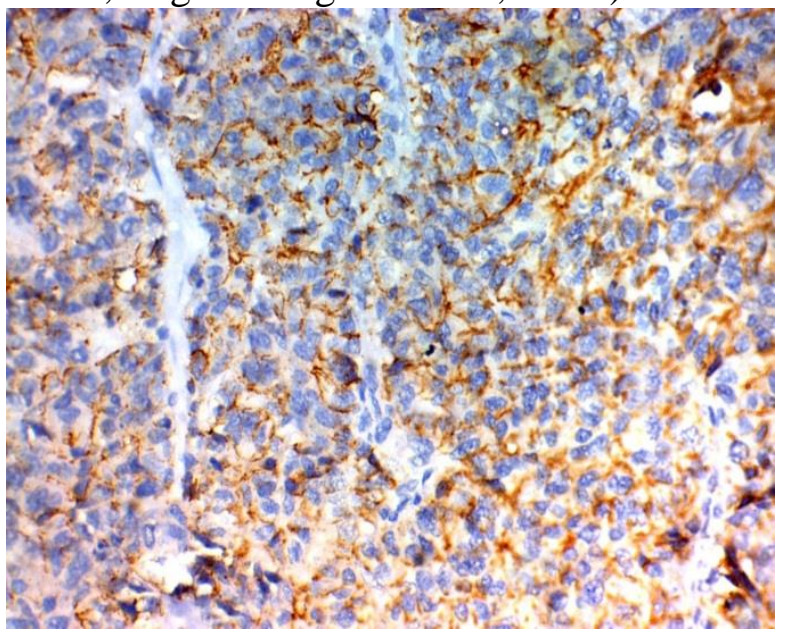

Figure 4 Colorectal adenocarcinoma grade III showing weak, incomplete linear membranous claudin 1 staining, score -7 (decreased expression) (Immunoperoxidase staining, DAB chromogen, Mayer's hematoxyline counter stain, original magnification, $\mathrm{X} 400$ ). 
Table 2 Correlation of claudin1 expression with clinicopathological characteristics of primary colorectal carcinoma cases

\begin{tabular}{|c|c|c|c|c|c|}
\hline \multirow[b]{2}{*}{ Characteristics } & \multirow[b]{2}{*}{$\mathrm{N}$} & \multicolumn{2}{|c|}{ Claudin1 expression } & \multirow[b]{2}{*}{$\chi^{2}$} & \multirow[b]{2}{*}{ p-value } \\
\hline & & $\begin{array}{l}\text { Similar } \\
(\mathrm{n}=14)\end{array}$ & $\begin{array}{c}\text { Decreased } \\
(\mathrm{n}=26)\end{array}$ & & \\
\hline \multicolumn{6}{|l|}{ Histological type } \\
\hline Adenocarcinoma (NOS) & 31 & $13(41.9 \%)$ & $18(58.1 \%)$ & \multirow{3}{*}{3.053} & \multirow{3}{*}{$\begin{array}{c}0.217 \\
\text { (NS) }\end{array}$} \\
\hline Mucinous & 7 & $1(14.3 \%)$ & $6(85.7 \%)$ & & \\
\hline Signet ring & 2 & $0(0 \%)$ & $2(100 \%)$ & & \\
\hline \multicolumn{6}{|l|}{ Tumor size } \\
\hline$\leq 5 \mathrm{~cm}$ & 27 & $9(33.3 \%)$ & $18(66.7 \%)$ & \multirow{2}{*}{$0.101 *$} & \multirow{2}{*}{$\begin{array}{l}1.000 \\
(\mathrm{NS})\end{array}$} \\
\hline$>5 \mathrm{~cm}$ & 13 & $5(38.5 \%)$ & $8(61.5 \%)$ & & \\
\hline \multicolumn{6}{|l|}{ Grading } \\
\hline Grade I & 17 & $10(58.8 \%)$ & $7(41.2 \%)$ & \multirow{3}{*}{7.801} & \multirow{3}{*}{$\begin{array}{c}0.020 \\
(\mathrm{~S})\end{array}$} \\
\hline Grade II & 13 & $3(23.1 \%)$ & $10(76.9 \%)$ & & \\
\hline Grade III & 10 & $1(10 \%)$ & $9(90 \%)$ & & \\
\hline \multicolumn{6}{|l|}{ Lymphovascular invasion } \\
\hline Absent & 28 & $14(50 \%)$ & $14(50 \%)$ & \multirow{2}{*}{$9.231 *$} & \multirow{2}{*}{$\begin{array}{l}0.003 \\
(\mathrm{HS})\end{array}$} \\
\hline Present & 12 & $0(0 \%)$ & $12(100 \%)$ & & \\
\hline \multicolumn{6}{|l|}{ Neural invasion } \\
\hline Absent & 35 & $14(40 \%)$ & $21(60 \%)$ & \multirow{2}{*}{$3.007 *$} & \multirow{2}{*}{$\begin{array}{l}0.143 \\
(\mathrm{NS})\end{array}$} \\
\hline Present & 5 & $0(0 \%)$ & $5(100 \%)$ & & \\
\hline \multicolumn{6}{|l|}{ Lymphocytic infiltration } \\
\hline Mild & 11 & $1(9.1 \%)$ & $10(90.9 \%)$ & \multirow{3}{*}{21.019} & \multirow{3}{*}{$\begin{array}{l}<0.001 \\
(\mathrm{HS})\end{array}$} \\
\hline Moderate & 18 & $3(16.7 \%)$ & $15(83.3 \%)$ & & \\
\hline Marked & 11 & $10(90.9 \%)$ & $1(9.1 \%)$ & & \\
\hline \multicolumn{6}{|l|}{ Peritumoral budding } \\
\hline Low & 17 & $13(76.5 \%)$ & $4(23.5 \%)$ & \multirow{2}{*}{22.350} & \multirow{2}{*}{$\begin{array}{c}<0.001 \\
(\mathrm{HS})\end{array}$} \\
\hline High & 23 & $1(4.3 \%)$ & $22(95.7 \%)$ & & \\
\hline \multicolumn{6}{|l|}{ Lymph node metastasis } \\
\hline Negative & 18 & $13(72.2 \%)$ & $5(27.8 \%)$ & \multirow{2}{*}{19.931} & \multirow{2}{*}{$\begin{array}{c}<0.001 \\
(\mathrm{HS})\end{array}$} \\
\hline Positive & 22 & $1(4.5 \%)$ & $21(95.5 \%)$ & & \\
\hline \multicolumn{6}{|l|}{$\overline{\text { Lymph node ratio }}$} \\
\hline LNR1 $(\leq 0.25)$ & 25 & $14(56 \%)$ & $11(44 \%)$ & & $<0.001$ \\
\hline LNR2 $(>0.25)$ & 15 & $0(0 \%)$ & $15(100 \%)$ & 12.923 & (HS) \\
\hline $\begin{array}{l}\chi^{2}: \text { Chi-square test. } \\
* \text { Fisher's exact test. } \\
\mathrm{p}<0.05 \text { is significant. }\end{array}$ & & $\begin{array}{l}\text { NS: Non signi } \\
\text { S: significant } \\
\text { HS: Highly si }\end{array}$ & ant & & \\
\hline
\end{tabular}


No statistically significant relationship was found between histological type and claudin 1 expression $\quad(\mathbf{p}=\mathbf{0 . 2 1 7})$. No statistically significant relationship was found between tumor size and claudin1 expression $(p=1.000)$. A statistically significant relationship was found between grading and claudin1 expression $(\mathbf{p}=\mathbf{0 . 0 2 0})$. There was a highly statistically significant difference considering the lymphovascular invasion and claudin1 expression $(\mathbf{p}=\mathbf{0 . 0 0 3})$. The association between neural invasion and claudin1 expression was not found to be statistically significant ( $\mathbf{p}=\mathbf{0 . 1 4 3})$. A highly statistically significant relationship was found between degree of lymphocytic infiltration, peritumoral budding, lymph node ratio, lymph node metastasis, Dukes staging and claudin1 expression ( $\mathbf{p}<\mathbf{0 . 0 0 1})$, Table 3.

Table 3 Correlation of claudin 1 expression with Dukes staging

\begin{tabular}{|c|c|c|c|c|c|}
\hline \multirow[b]{2}{*}{ Dukes staging } & \multirow[b]{2}{*}{$\mathrm{N}$} & \multicolumn{2}{|c|}{ Claudin1 expression } & \multirow[b]{2}{*}{$\chi^{2}$} & \multirow[b]{2}{*}{ p-value } \\
\hline & & $\begin{array}{l}\text { Similar } \\
(\mathrm{n}=14)\end{array}$ & $\begin{array}{c}\text { Decreased } \\
(\mathrm{n}=26)\end{array}$ & & \\
\hline Stage A & 3 & $3(100 \%)$ & $0(0 \%)$ & \multirow{4}{*}{21.197} & \multirow{4}{*}{$\begin{array}{c}<0.001 \\
\text { (HS) }\end{array}$} \\
\hline Stage B & 15 & $10(66.7 \%)$ & $5(33.3 \%)$ & & \\
\hline Stage C & 18 & $1(5.6 \%)$ & $17(94.4 \%)$ & & \\
\hline Stage D & 4 & $0(0 \%)$ & $4(100 \%)$ & & \\
\hline
\end{tabular}

$\chi 2$ : Chi-square test. HS: Highly significant

$\mathrm{p}<0.05$ is significant.

\section{S100A4 expression}

Normal colonic and rectal mucosa showed negative staining for S100A4 (Figure 5). Ten tumors showed absent (score 0-2), or only mild (score 3) (Figure 6), nine tumors showed moderate S100A4 expression (score 4-5) (Figure 7) and seven tumors showed marked S100A4 expression (score 6) (Figure 8). Most of the grade I tumors (11/17) (64.7\%) showed negative S100A4 expression, whereas all the grade II and III tumors (100\%) showed positive S100A4 expression. There was a statistically significant difference between the histological type and S100A4 expression ( $\mathbf{p}=$ 0.044). No statistically significant relationship was found between tumor size and claudin 1 expression ( $\mathbf{p}=\mathbf{0 . 6 9 7})$.

A highly statistically significant relationship was found between grade of differentiation and S100A4 expression (p =0.002). A highly statistically significant relationship was found between the lymphovascular invasion $(\mathbf{p = 0 . 0 0 2})$, degree of lymphocytic infiltration, peritumoral budding, lymph node metastasis, lymph node ratio and S100A4 expression (p <0.001) (Table 4). S100A4 showed high specific association with tumor staging. There was a highly statistically significant relationship was found between Dukes staging and S100A4 expression $(\mathbf{p}<\mathbf{0 . 0 0 1})$ (Table 5). 


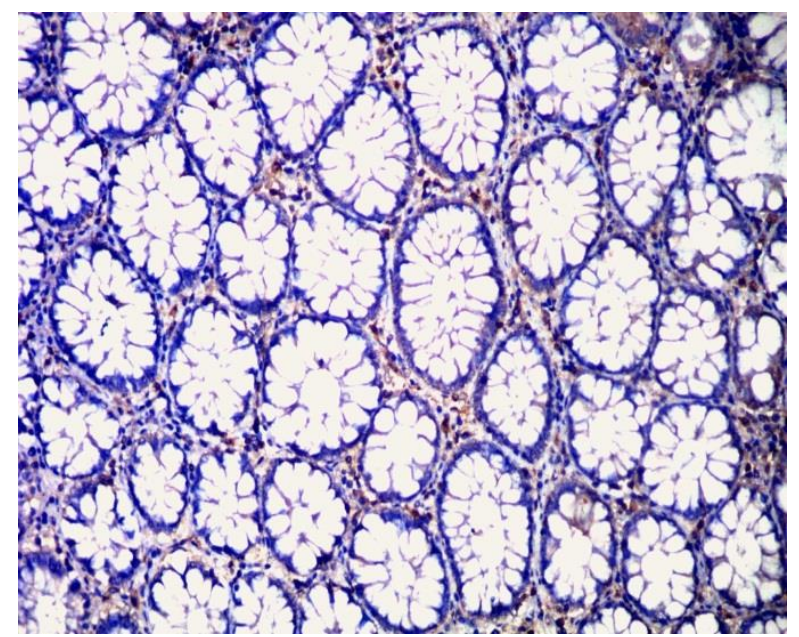

Figure 5 Normal colorectal mucosa showing negative staining for S100A4 (Immunoperoxidase staining, DAB chromogen, Mayer's hematoxyline counter stain, original magnification X400).

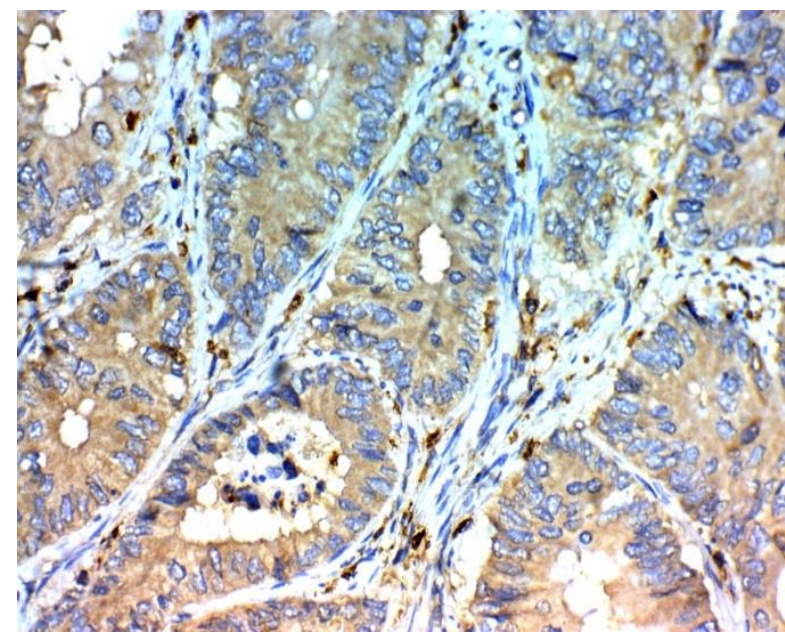

Figure 6 Colorectal adenocarcinoma, grade I showing S100A4 mild staining (score 3) (Immunoperoxidase staining, DAB chromogen, Mayer's hematoxyline counter stain, original magnification X400).

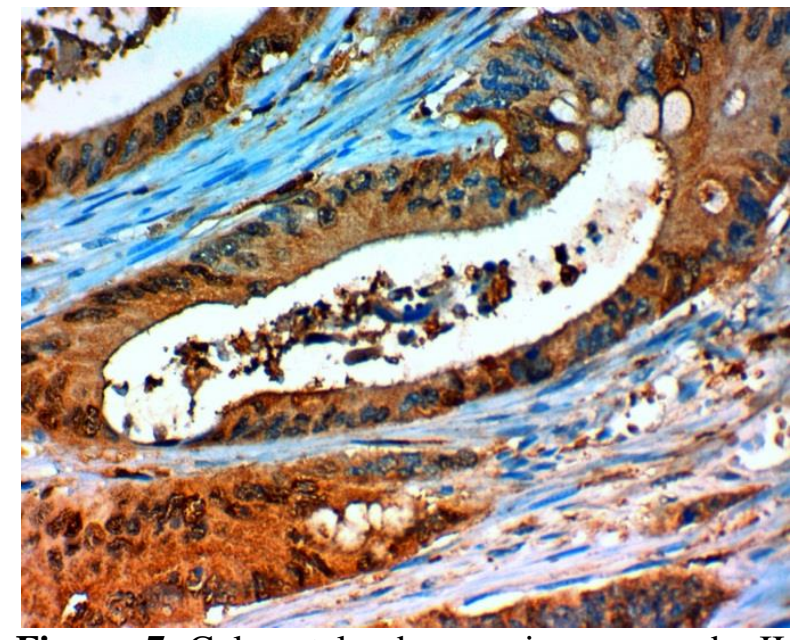

Figure 7 Colorectal adenocarcinoma, grade II showing S100A4 moderate staining (score 5) (Immunoperoxidase staining, DAB chromogen, Mayer's hematoxyline counter stain, original magnification X400).

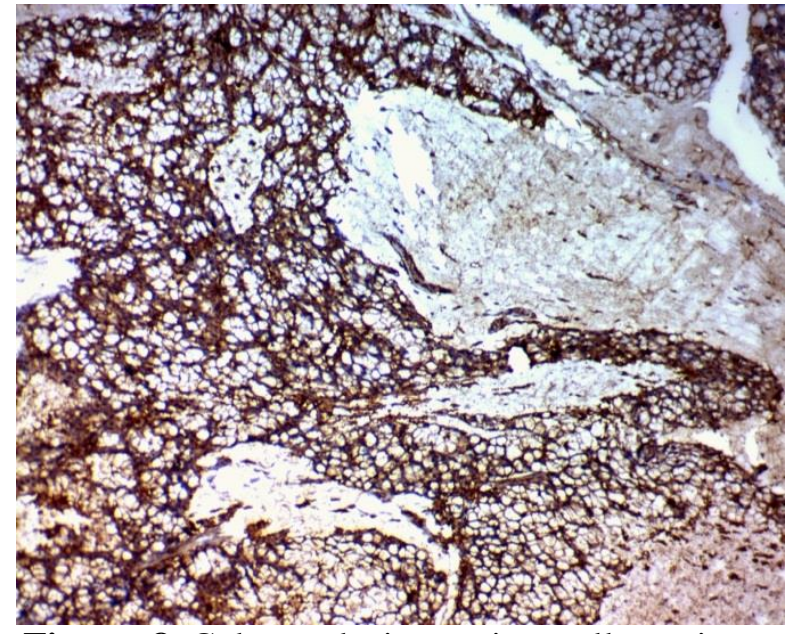

Figure 8 Colorectal signet ring cell carcinoma showing S100A4 strong staining (score 6) (Immunoperoxidase staining, DAB chromogen, Mayer's hematoxyline counter stain, original magnification X200). 
Table 4 Correlation of S100A4 expression with clinicopathological characteristics of primary colorectal carcinoma cases

\begin{tabular}{|c|c|c|c|c|c|}
\hline \multirow[b]{2}{*}{ characteristics } & \multirow[b]{2}{*}{$\mathrm{N}$} & \multicolumn{2}{|c|}{ S100A4 expression } & \multirow[b]{2}{*}{$x^{2}$} & \multirow[b]{2}{*}{ p-value } \\
\hline & & $\begin{array}{c}\text { Negative } \\
\text { expression } \\
(\mathrm{n}=14)\end{array}$ & $\begin{array}{l}\text { Positive } \\
\text { expression } \\
(\mathrm{n}=26)\end{array}$ & & \\
\hline \multicolumn{6}{|l|}{ Histological type } \\
\hline Adenocarcinoma (NOS) & 31 & $14(45.2 \%)$ & $17(54.8 \%)$ & \multirow{3}{*}{6.253} & \multirow{3}{*}{$\begin{array}{c}0.044 \\
(\mathrm{~S})\end{array}$} \\
\hline Mucinous & 7 & $0(0 \%)$ & $7(100 \%)$ & & \\
\hline Signet ring & 2 & 0 & $2(100 \%)$ & & \\
\hline \multicolumn{6}{|l|}{ Tumor size } \\
\hline$\leq 5 \mathrm{~cm}$ & 27 & $10(37 \%)$ & $17(63 \%)$ & \multirow{2}{*}{0.152} & \multirow{2}{*}{$\begin{array}{c}0.697 \\
(\mathrm{NS})\end{array}$} \\
\hline$>5 \mathrm{~cm}$ & 13 & $4(30.8 \%)$ & $9(69.2 \%)$ & & \\
\hline \multicolumn{6}{|l|}{ Histological type } \\
\hline Adenocarcinoma (NOS) & 31 & $14(45.2 \%)$ & $17(54.8 \%)$ & \multirow{3}{*}{6.253} & \multirow{3}{*}{$\begin{array}{c}0.044 \\
(\mathrm{~S})\end{array}$} \\
\hline Mucinous & 7 & $0(0 \%)$ & $7(100 \%)$ & & \\
\hline Signet ring & 2 & $0(0 \%)$ & $2(100 \%)$ & & \\
\hline \multicolumn{6}{|l|}{ Grading } \\
\hline Grade I & 17 & $11(64.7 \%)$ & $6(35.3 \%)$ & \multirow{3}{*}{12.791} & \multirow{3}{*}{$\begin{array}{c}0.002 \\
(\mathrm{HS})\end{array}$} \\
\hline Grade II & 13 & $3(23.1 \%)$ & $10(76.9 \%)$ & & \\
\hline Grade III & 10 & $0(0 \%)$ & $10(100 \%)$ & & \\
\hline \multicolumn{6}{|l|}{ Lymphovascular invasion } \\
\hline Absent & 28 & $14(50 \%)$ & $14(50 \%)$ & \multirow{2}{*}{9.231} & \multirow{2}{*}{$\begin{array}{c}0.002 \\
\text { (HS) }\end{array}$} \\
\hline Present & 12 & $0(0 \%)$ & $12(100 \%)$ & & \\
\hline \multicolumn{6}{|l|}{ Neural invasion } \\
\hline Absent & 35 & $14(40 \%)$ & $21(60 \%)$ & \multirow{2}{*}{3.077} & \multirow{2}{*}{$\begin{array}{c}0.079 \\
(\mathrm{NS})\end{array}$} \\
\hline Present & 5 & $0(0 \%)$ & $5(100 \%)$ & & \\
\hline \multicolumn{6}{|l|}{ Lymphocytic infiltration } \\
\hline Mild & 11 & $0(0 \%)$ & $11(100 \%)$ & \multirow{3}{*}{22.329} & \multirow{3}{*}{$\begin{array}{c}<0.001 \\
(\mathrm{HS})\end{array}$} \\
\hline Moderate & 18 & $4(22.2 \%)$ & $14(77.8 \%)$ & & \\
\hline Marked & 11 & $10(90.9 \%)$ & $1(9.1 \%)$ & & \\
\hline \multicolumn{6}{|l|}{ peritumoral budding } \\
\hline Low & 17 & $14(82.4 \%)$ & $3(17.6 \%)$ & \multirow{2}{*}{29.140} & $<0.001$ \\
\hline High & 23 & $0(0 \%)$ & $23(100 \%)$ & & $(\mathrm{HS})$ \\
\hline Lymph node metastasis & & & & & \\
\hline Negative & 18 & $14(77.8 \%)$ & $4(22.2 \%)$ & 26325 & $<0.001$ \\
\hline Positive & 22 & $0(0 \%)$ & $22(100 \%)$ & 20.525 & $(\mathrm{HS})$ \\
\hline Lymph node ratio & & & & & \\
\hline LNR1 $(\leq 0.25)$ & 25 & $14(56 \%)$ & $11(44 \%)$ & 12923 & $<0.001$ \\
\hline LNR2 (>0.25) & 15 & $0(0 \%)$ & $15(100 \%)$ & 12.923 & $(\mathrm{HS})$ \\
\hline
\end{tabular}

\begin{tabular}{lcc}
\hline$\chi 2:$ Chi-square test. & NS: Non significant & S: significant \\
$\mathrm{p}<0.05$ is significant. & HS: Highly significant
\end{tabular}

Table 5 Correlation of S100A4 expression with Dukes staging.

\begin{tabular}{|c|c|c|c|c|c|}
\hline \multirow[b]{2}{*}{ Dukes staging } & \multirow[b]{2}{*}{$\mathrm{N}$} & \multicolumn{2}{|c|}{ S100A4 expression } & \multirow[b]{2}{*}{$\chi^{2}$} & \multirow[b]{2}{*}{$\mathrm{p}$ - value } \\
\hline & & $\begin{array}{c}\text { Negative expression } \\
(\mathrm{n}=14)\end{array}$ & $\begin{array}{c}\text { Positive expression } \\
(\mathrm{n}=26)\end{array}$ & & \\
\hline Stage A & 3 & $3(100) \%$ & $0(0 \%)$ & \multirow{4}{*}{27.106} & \multirow{4}{*}{$\begin{array}{c}<0.001 \\
(\mathrm{HS})\end{array}$} \\
\hline Stage B & 15 & $11(73.3) \%$ & $4(26.7 \%)$ & & \\
\hline Stage C & 18 & $0(0 \%)$ & $18(100 \%)$ & & \\
\hline Stage D & 4 & $0(0 \%)$ & $4(100 \%)$ & & \\
\hline
\end{tabular}

\section{Concordance between S100A4 and claudin1 staining results}




\section{Table 6 Correlation of claudin 1 expression with S100A4 expression}

\begin{tabular}{|c|c|c|c|c|c|c|c|}
\hline & \multirow{3}{*}{$\mathrm{N}$} & \multicolumn{4}{|c|}{ S100A4 expression } & \multirow{3}{*}{$\mathrm{r}$} & \multirow{3}{*}{ p-value } \\
\hline & & \multicolumn{2}{|c|}{$\begin{array}{l}\text { Negative } \\
(\mathrm{n}=14)\end{array}$} & \multicolumn{2}{|c|}{$\begin{array}{c}\text { Positive } \\
(\mathrm{n}=26)\end{array}$} & & \\
\hline & & $\overline{\text { No }}$ & $\%$ & No & $\%$ & & \\
\hline \multicolumn{8}{|c|}{ Claudin1 expression } \\
\hline Similar & 14 & 12 & $85.7 \%$ & 2 & $14.3 \%$ & \multirow{2}{*}{$-0.8^{*}$} & \multirow{2}{*}{$\begin{array}{c}0.0 \\
(\mathrm{HS})\end{array}$} \\
\hline Decreased & 26 & 2 & $7.7 \%$ & 24 & $92.3 \%$ & & \\
\hline
\end{tabular}

r: Pearson's correlation coefficient.

$\mathrm{p}<0.05$ is significant.

There is a highly statistically significant inverse correlation between claudin 1 expression and S100A4 expression; when claudin1 expression decreases, S100A4 expression becomes positive.

\section{DISCUSSION}

Early detection of tumorigenesis and metastases is critical to improving treatment strategies and patient outcomes. The development of reliable biomarkers that can be routinely applicable for early detection of progression and prognosis is strongly needed. (20)

In view of mean age at diagnosis, data from this study reveals younger age at diagnosis (49.68 \pm 11.39 ), than reported in the wide literature. In these, the mean age was found to be $57.9 \pm 11.3^{(\mathbf{2 1})}, 62.1 \pm 13.8^{\text {(22) }}$, $65.7 \pm 12.5^{\text {(23) }}, 68.5 \pm 11.1$ (24) Schistosomal $^{\text {(24) }}$ colitis is more commonly associated with earlier onset multicenteric CRC. ${ }^{(25)}$ In our study only one case of bilharziasis was documented. Rare findings of bilharziasis in this study may be attributed to different demographic areas from which the specimens were collected.

In the present study the commonest histologic type detected was adenocarcinoma (NOS) $(77.5 \%)$. This was in agreement with the findings given by Süren et al. ${ }^{(22)}$ and Resnick et al. ${ }^{(26)}$, who reported that $81.4 \%$ and $80.6 \%$ of their cases were diagnosed as adenocarcinoma (NOS), respectively. A higher percentage $(93.1 \%)$ of adenocarcinoma cases (NOS) was reported by Niu et al. ${ }^{(\mathbf{1 9})}$, while Mansour and Hennis ${ }^{(21)}$ reported a lower percentage $(60 \%)$ of adenocarcinoma cases (NOS).

In the present study mucinous and signetring cell carcinoma represented $17.5 \%$ and $5 \%$ of the studied cases. This percentage was in line with the results of Süren et al. ${ }^{(22)}$, who reported that $14.3 \%$ and $4.3 \%$ of the cases diagnosed as mucinous and signet -ring cell carcinoma respectively. While, $\mathrm{Xu}$ et al. ${ }^{(27)}$, reported a higher percentage of mucinous $(33.8 \%)$ and signet ring cell carcinoma $(17.6 \%)$.

Regarding the grading of colorectal carcinoma, the present study showed that grade I was more encountered than other grades, representing $42.5 \%$, and $32.5 \%$ of cases were diagnosed as grade II and $25 \%$ were diagnosed as grade III. These results are in agreement with those of Abdelzaher et al. ${ }^{(28)}$, which reported an incidence of $(46 \%)$. But higher percentage was reported by Süren et al. ${ }^{(22)}(65.7 \%)$ and Kinugasa et al. (23) $(60 \%)$. On the other hand, Niu et al. ${ }^{(19)}$ and Abdelzaher et al. ${ }^{(27)}$ found that the commonest grade was grade II representing $61.8 \%$ and $41.2 \%$ respectively. Mansour and Hennis ${ }^{(21)}$ found that the commonest grade was grade III representing $46.7 \%$ of the cases.

According to staging, the mostly diagnosed cases were at stage Dukes C /TNM III (45\%), which in line with those of Süren et al. ${ }^{(22)}$, (41.4\%). On contrary Lee et al. ${ }^{(8)}$, found that stage II TNM (42.9\%) was the mostly diagnosed stage. Kwak et al. ${ }^{(29)}$ and Kinugasa et al. ${ }^{(\mathbf{2 3})}$ reported equal percentage of cases at stages B\&C $(33.3 \%, 41 \%)$ respectively.

There is no a clear difference between Dukes staging and TNM staging and the strength of agreement is strong. The Dukes staging is simple and easy applicable. (30) However, several subgroups of TNM (IIB, IIC and IVB) are not presented.

Several studies analyzed the roles of tight junctions in oncogenesis. Suppression of 
claudin-1 expression in colorectal cancer compared to normal mucosa and linked with depth of invasion, histological grade and lymphovascular invasion. ${ }^{(22,28)}$

Other studies on the other hand, have shown increased expression of claudin- 1 in CRC cells. ${ }^{(31,24)}$

Claudin1 expression was found to be progressively decreased among different grades, this finding is in line with those of $\mathrm{Al}$ Kattan et al. ${ }^{(31)}$, Süren et al. ${ }^{(22)}$, Mansour and Hennis $^{(21)}$, Abdelzaher et al. ${ }^{(28)}$ and Resnick et al. ${ }^{(26)}$.

On the contrary Nakagawa et al. ${ }^{(32)}$, found that there was no loss of claudin1 expression in high grade tumors. This discrepancy might be attributed to the difference in percentage of poorly differentiated tumors in the present study $(25 \%)$ and in the other study $(7.5 \%)$.

No loss of claudin 1 expression was noticed in a case of mucinous carcinoma (grade III). Several studies reported that some of poorly differentiated tumors have high degree of microsatellite instability (MSI-H) proved by molecular testing and behave as low grade. ${ }^{(33)}$

Claudin 1 expression was not found to be related to tumor types. This finding is supported by studies of Süren et al. ${ }^{(22)}$, Mansour and Hennis ${ }^{(21)}$, Abdelzaher et al. ${ }^{(28)}$, and Resnick et al. ${ }^{(\mathbf{2 6})}$.

Similar to a study done by Nakagawa et al. ${ }^{(32)}$, it was found that claudin1 expression was not affected with an increase in tumor size.

In the present study neural invasion was not associated with decreased claudin 1 expression was not found to be statistically significant in contrast to the findings of Süren et al. ${ }^{(22)}$; this may be due to small number of detected tumors which had neural invasion in our study.

Lymphovascular invasion was associated with decreased claudin 1 expression and this is consistent with the results obtained by Nakagawa et al. ${ }^{(32)}$, but on the contrary, Resnick et al. ${ }^{(26)}$ found that there was no relation between claudin1 expression and Lymphovascular invasion.

Marked lymphocytic infiltration was found to be associated with no loss of claudin1 expression. This is in keeping with observations of Süren et al. ${ }^{\text {(22) }}$. This finding suggests that decreased claudin 1 expression is associated with poor immune response.

Consistent with the finding of this study, claudin1 expression was significantly decreased in lymph node positive cases in several studies ${ }^{(\mathbf{2 2 , 3 1 , 3 2 )}}$. On the contrary Huo et al. ${ }^{(24)}$, and Kinugasa et al. $^{(23)}$, noticed an increase in claudin-1 expression associated with metastatic lymph nodes.

Peritumoral budding is a putative hallmark of CRC cell invasion and has previously been shown to be associated with various clinicopathological parameters including lymph node metastasis, lymphovascular invasion, distant metastasis, local recurrence and poor outcome. ${ }^{(\mathbf{3 4})}$ Our results showed decreased expression of claudin 1 at the invasive margin of CRC.

In the present study, high LNR associated with decreased claudin1 expression. As far as we know, this is the first study that correlates claudin 1 expression with lymph node ratio.

In line with our results, several studies Süren et al. ${ }^{(22)}$, Mansour and Hennis ${ }^{(21)}$, Abdelzaher et al. ${ }^{(\mathbf{2 8})}$, and Resnick et al. ${ }^{(\mathbf{2 6})}$ confirmed the observation that decreased claudin1 expression was associated with progression of tumor staging. In contrast, Gröne et al. ${ }^{(35)}$ reported no loss of claudin 1 at advanced stage.

S100A4, a metastasis-related molecule which implicated in the regulation of angiogenesis, motility and invasion, has a role in EMT process. ${ }^{(\mathbf{5})}$

The present study demonstrated that $65 \%$ of cases were S100A4 positive. This finding is close to those of Niu et al. ${ }^{(\mathbf{1 9})}$, Huang et al. ${ }^{(36)}$, and $\mathrm{Xu}$ et al. ${ }^{(27)}$ who reported $54.2 \%$, $50.9 \%$ and $61.8 \%$ respectively. Lower percentage was reported by Lee et al. ${ }^{(\mathbf{8})}$, (23.1\%) and Kwak et al. ${ }^{\text {(29) }}$ (33.1\%).

S100A4 overexpression was found to be related to histological type, in contrary to Niu et al. ${ }^{(\mathbf{1 9})}$ who didn't find any correlation.

Similar to our findings Niu et al. ${ }^{(19)}$ and Huang et al. ${ }^{(36)}$ found that tumor size not related to S100A4 overexpression.

In the present study, higher tumor grade is associated with S100A4 overexpression, 
this finding in contrast to that of Kwak et al. ${ }^{(29)}$

Lymphovascular invasion was associated with S100A4 overexpression; this is consistent with the finding of Lee et al. ${ }^{(\mathbf{8})}$

Neural invasion was not found to be related to S100A4 overexpression, this disagree with the results done by Lee et al. ${ }^{(\mathbf{8})}$ who found that neural invasion is associated with S100A4 overexpression. This discrepancy may be related to different number of cases between the present study (40) cases and his study (333) cases.

Our results suggest an overexpression of S100A4 at the invasive margin of CRC; these results are in agreement with those of Lee et al. $^{(8)}$

Mild lymphocytic infiltration found to be associated with S100A4 overexpression, which is close to results done by Lee et al. ${ }^{(\mathbf{8})}$

It was found that lymph node metastasis is associated with S100A4 overexpression, This finding is in agreement with that of Niu et al. ${ }^{(\mathbf{1 9})}$, Huang et al. ${ }^{(36)}$ and $\mathrm{Xu}$ et al. ${ }^{(27)}$, but not in line with that of ${ }^{(31)}$, who reported no relationship between lymph node metastasis and S100A4 overexpression.

As regards to lymph node ratio, it was found that high LNR is associated with S100A4 overexpression, which is in agreement of that of Lee et al. ${ }^{(8)}$

Similar to the findings of Niu et al. ${ }^{(19)}$, Huang et al. ${ }^{(36)}$ and $\mathrm{Xu}$ et al. ${ }^{(27)}$ S100A4 overexpression was related to progression of tumor staging. S100A4 overexpression is associated with increased ability to invade and metastasize as well as the poor survival of patients ${ }^{(37)}$. On the contrary, Kwak et al. ${ }^{(29)}$, reported no relationship between advanced stage and S100A4 overexpression.

As far as we know, this is the first study that made a correlation between claudin 1 and S100A4 expression in primary CRC. There is an inverse correlation between claudin 1 and S100A4 expression which means that loss of tight junctions and acquisition of mesenchymal properties lead to EMT and subsequently into invasion and metastasis.

\section{CONCLUSIONS}

Loss of tight junctions represented by decreased claudin 1 expression and acquisition of mesenchymal properties represented by
S100A4 overexpression are important for primary colorectal carcinoma progression and metastasis. Decreased claudin1 represent an initial step in EMT and overexpression of S100A4 represents a late step of EMT, which resulted in invasion and metastasis. So, claudin1 and S100A4 might be used as prognostic markers for tumor progression in primary CRC.

\section{RECOMMENDATIONS}

If the lymph node ratio is to have excellent prognostic value, a systematical evaluation involving a large scale study is needed to establish a more reliable cut off value.

An enhanced understanding of molecular basis of colorectal carcinoma, especially hereditary nonpolyposis syndrome and microsatellite instability will aid the design of screening program for identification of many individuals at high risk for developing colorectal carcinoma.

More detailed studies in a large cohort would be useful to support the proposal of closeness of bilharzizsis with colorectal carcinoma.

Abbreviations: CRC: colorectal carcinoma. EMT:epithelial mesencymal transition, LNR: lymph node ratio.

Acknowledgements: There is no funding. Conflicts of interests: The authors declare that they have no conflicts of interests.

\section{REFERENCES}

1- Jemal A., Siegel R., Ward E. et al.,: Cancer statistics. Cancer JClin; 2008;58:71-96.

2- El-Bolkainy N., Nouh M. and El-Bolkainy T.: Topographic pathology of cancer. 3rd ed. Cairo: Egyptian National Cancer Institute; 2005;p: 83.

3- Mazzuccheli L.: Protein S100A4: too long overloked by pathol-ogists? Am J Pathol; 2002;160: 7-13.

4- Stein U., Arlt F., Walther W., et al.,: The metastasis-associated gene S100A4 is a novel target of beta-catenin/T-cell factor signaling in colon cancer. Gastroenterology; 2006; 131 : 1486-1500.

5- Mishra S., Siddique H. and Saleem M.: S100A4 calcium-binding protein is key player in tumor progression and metastasis: preclinical and clinical evidence. Cancer Metastasis Rev; 2012;31: 163-172.

6- Mencia N., Selga E., Rico I., et al.,: Overexpression of S100A4 in human cancer 
cell lines resistant to methotrexate. BMC Cancer 2010;10: 250.

7- Kang Y., Jung C., Lee A. et al.,: Prognostic significance of S100A4 mRNA and protein expression in colorectal cancer. Journal of SurgicalOncology; 2012; 105:119-124.

8- Lee S., Choi S., Kim W., et al.,: Combined aberrant expression of E-cadherin and S100A4, but not beta-catenin is associated with disease-free survival and overall survival in colorectal cancer patients. DiagnPathol 2013, 8(1):99.

9- Boye K. and Maelandsmo G.: S100A4 and metastasis: a small actor playing many roles. Am J Pathol; 2010;176:528-535.

10-Stebbing J., Filipović A. and Giamas G.: Claudin-1 as a promoter of EMT in hepatocellular carcinoma Oncogene 2013;32, 4871-4872.

11-Ersoz S., Mungan S., Cobanoglu U. et al.,: Prognostic importance of claudin-1 and claudin-4 expression in colon carcinomas. Pathol Res Pract; 2011;207(5):285-9.

12-Hamilton S., Bosman F., Boffetta P., et al.,: Carcinoma of the colon and rectum. In: Bosman F., Carneiro F., Hruban R., Theise N., editors. WHO Classification of Tumors of the Digestive System. 4th ed. Lyon: IARC; 2010;134-146.

13-Petras R. and Frankel W.: Large intestine (colon). In: Weidner N, Cote RJ, Suster S, and Weiss LM (eds): Modern surgical pathology, second edition, volume 1 . Saunders Elsevier, China. P. 2009;756765,768 -773.

14-Dukes Bussey H., Lamb J., et al.,: The examination and classification of operation specimens of intestinal cancer. Bull Int Assoc Med Mus; 1947;27:55-65.

15-Ueno H.,Mochizuki H.,Hashiguchi Y., et al.,: Risk factors for an adverse outcome in early invasive colorectalcarcinoma.Gastroenterology; 2004; 127:385-94.

16-Shinto E., Mochizuki H., Ueno H., et al.,: A novel classification of tumor budding in colorectal cancer based on the presence of cytoplasmic pseudofragments around budding foci. Histopathology; 2005;47:2531.

17-Park I., Yu C., Lim S., et al.,: Ratio of metastatic lymph nodes is more important for rectal cancer patients treated with preoperative Chemoradiotherapy. World J Gastroenterol; 2015;21(11): 3274-3281.
18- Sheehan G., Kallakury B., Sheehan C., et al.,: Loss of claudins-1 and -7 andexpression of claudins-3 and -4 correlate with prognostic variablesin prostatic adenocarcinomas. Hum Pathol; 2007;38(4):564-9.

19-NiuY., Wang L., Cheng C., et al.,: Increased expressions of SATB1 and S100A4 are associated with poor prognosis in human colorectal carcinoma. APMIS; 2014;1-9.

20-Cao H., Xu E., Liu H., et al.,: Epithelial mesenchymal transition in colorectal cancer metastasis: A system review. PathologyResearch and Practice; 2015;211(8):557-569.

21-Mansour S. and Hennis M.: Study on the expression of claudin-1, E-cadherin, and $\mathrm{Ki}$ 67 and their clinical significance in colon cancer. Egyptian Journal of Pathology; Vol 2012;32 (2): 238-244.

22- Süren D., Yildirim M., Kaya V., et al.,: Loss of tight junction proteins (Claudin 1, 4, and 7) correlates with aggressive behavior in colorectal carcinoma. Med Sci Monit; 2014;20: 1255-1262.

23- Kinugasa T, Huo Q., Higashi D., et al.,: Selective Up-regulation of Claudin-1 and Claudin-2 in Colorectal Cancer. ANTICANCER RESEARCH 2007;27: 3729-3734.

24- Huo Q., Kinuugasa T., Wang L., et al.,: Claudin-1 Protein is a Major Factor Involved in the Tumorigenesis of Colorectal Cancer. ANTICANCER RESEARCH 2009;29: 851858.

25- Madbouly K., Senagore A., Mukerjee A., et al.,: Colorectal cancer in a population with endemic Schistosoma mansoni: is this an atrisk population? Int $\mathrm{J}$ Colorectal Dis 2007;22: 175-81.

26-Resnick M., Konkin T., Routhier J., et al.,: Claudin-1 is a strong prognostic indicator in stage II colonic cancer: a tissue microarray study. Mod. Pathol. 2005;18, 511-518.

27- Xu X., Lu L., Song L., et al.,: Expression and Significance of Protein S100A4 in Colorectal Carcinoma. U.S. Chinese Journal of Lymphology and Oncology. Volume 2007;6(4): 167-172.

28-Abdelzaher E., Rizk A., Bessa S., et al.,: Predictive value of immunohistochemical expression of claudin-1 in colonic carcinoma. Journal of the Egyptian National Cancer Institute; 2011;23: 123-131.

29-Kwak J., Lee H., Kim., et al.,: Expression of protein S100A4 is a predictor of recurrence in colorectal cancer. World $J$ Gastroenterol ; 2010; 16(31): 3897-3904. 
30- Compton C. and Greene F.: The staging of colorectal cancer. J Clin oncology; 2004;54(6): 295-308.

31-Al Kattan W., Ouban A., Nawab R., et al.,: Characterization and analysis of claudin-1 expression in colorectal cancer and its metastases: A pilot study; Integr Mol Med, 2015;2(4): 243-247

32-Nakagawa S., Miyoshi N. , Ishii H., et al.,: Expression of CLDN1 in colorectal cancer: A novel marker for prognosis. INTERNATIONAL JOURNAL OF ONCOLOGY 2011;39: 791-796.

33- Schneider R., Schneider C., Kloor M., et al.,: Lynch syndrome: clinical, pathological, and genetic insights. LangenbecksArch Surg; 2012;397:513-525.

34-Wang L., Kevans D., Mulcahy H., et al.,: Tumor budding is a strong and reproducible prognostic marker in T3N0 colorectal cancer. Am J SurgPathol; 2009;33:134.

35- Gröne J., Weber B., Staub E., et al.,: Differential expression of genes encoding tight junction proteins in colonic cancer: frequent dysregulation of claudin-1,-8 and12. Int J Colon Dis; 2007;22(6):651-9.

36-Huang L., Xu Y., Cai G., et al.,: S100A4 over-expression underlies lymph node metastasis and poor prognosis in colorectal cancer. World J Gastroenterol; 2011;17(1): 69-78.

37- Sack U., Walther W., Scudiero D., et al.,: Novel effect of antihelminthic Niclosamide on S100A4-mediated metastatic progression in colon cancer. J Natl Cancer Inst; 2011;103:1018-36. 